\title{
Hypersignal vasculaire ou « signe du spaghetti » en IRM séquence Flair : un signe précoce d'accident vasculaire cérébral ischémique
}

\section{Hyperintense Vessel or "Spaghetti Sign" on MRI Flair Images: an Early Sign of Acute Ischemic Stroke}

\section{H. Andrianjafy • L. Wajzer}

Reçu le 21 octobre 2013; accepté le 10 janvier 2014

(C) SFMU et Springer-Verlag France 2014

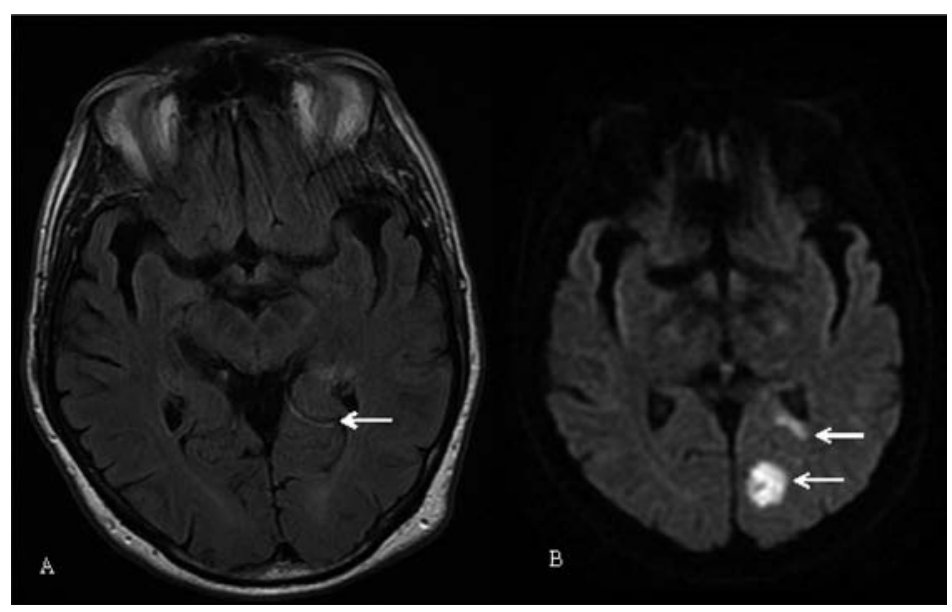

Fig. 1 A. IRM séquence Flair : hypersignal vasculaire de l'ACP gauche (flèche blanche)

B. IRM séquence de diffusion : AVC ischémique temporal interne et occipital gauche (flèches blanches)

Un homme de 63 ans est vu aux urgences suite à une lipothymie au volant de son véhicule. L'examen neurologique retrouve une anomie sur les noms propres et une hémianopsie latérale homonyme droite. Le score NIHSS est côté à 2 . Une IRM cérébrale est faite en urgence. La séquence de diffusion est normale, et la séquence Flair révèle un hypersignal vasculaire au niveau de l'artère cérébrale postérieure (ACP) gauche (Fig. 1A). Aucune occlusion vasculaire n'est visible sur l'ARM du polygone de Willis. Le patient est transféré en unité de soins intensifs neurovasculaire (USINV), et une nouvelle IRM cérébrale le lendemain confirme le diagnostic d'accident vasculaire cérébral (AVC) ischémique intéressant le territoire de l'ACP gauche (temporal interne et occipital gauche) (Fig. 1B).

\footnotetext{
H. Andrianjafy $\cdot$ L. Wajzer $(\bowtie)$

Service des urgences, Centre hospitalier Longjumeau,

159, rue du président François Mitterrand,

F-91160 Longjumeau, France

e-mail : hery.andrianjafy@ch-longjumeau.fr
}

Un hypersignal vasculaire cérébral en séquence Flair ou « signe du spaghetti » est un marqueur de bas débit artériel, qui signe un état circulatoire précaire dans le territoire irrigué, avec risque d'aggravation secondaire $[1,2]$. Cette anomalie peut être visible précocement après la survenue des signes cliniques d'AVC, et est particulièrement intéressante lorsque l'ARM du polygone de Willis n'est pas réalisée ou ininterprétable, notamment chez un patient agité. Ce signe doit alors conduire à transférer rapidement le patient en USINV.

\section{Références}

1. Sanossian N, Srave JL, Alger JR, et al (2009) Angiography reveals that fluid-attenuated inversion recovery vascular hyperintensities are due to slow flow, not thrombus. Am J Neuroradiol 30:564-68

2. Sanossian N, Hao Q, Liebeskind DS (2011) The thrombus and discontinuity of FLAIR vascular hyperintensity. Arch Neurol 68:950-1 